\title{
ORIGINAL ARTICLE Paediatric ART outcomes in a decentralised model of care in Cape Town, South Africa
}

M M Morsheimer, ${ }^{1}$ MD; A Dramowski, ${ }^{2}$ MB ChB, MMed, Cert Paed ID; H Rabie, ${ }^{2}$ MB ChB, MMed, Cert Paed ID; M F Cotton, ${ }^{2} \mathrm{MD}, \mathrm{PhD}$

\author{
${ }^{1}$ Division of Allergy and Immunology, The Children's Hospital of Philadelphia, USA \\ ${ }^{2}$ Division of Paediatric Infectious Diseases, Department of Paediatrics and Child Health, Stellenbosch University, \\ Cape Town, South Africa
}

Corresponding author: A Dramowski(dramowski@sun.ac.za)

\begin{abstract}
Background. Although subSaharan Africa faces the world's largest paediatric HIV epidemic, only 1 in 4 children has access to combination antiretroviral therapy (ART). A decentralised approach to HIV care is advocated, but programmes in resourcelimited settings encounter many challenges to community-initiated paediatric ART implementation.

Methods. A retrospective cohort analysis of 613 children receiving ART between 2004 and 2009 was performed in seven physician-run primary healthcare (PHC) clinics in Cape Town. Baseline characteristics, serial CD4+, viral load (VL) levels and status at study closure were collected.

Results. Two subgroups were identified: children who were initiated on ART in a PHC clinic $(n=343)$ and children who were down-referred from tertiary hospitals $(n=270)$. The numbers of children initiated on ART in PHC increased sevenfold over the study period. Down-referred children were severely ill at ART initiation, with higher VLs, lower CD4 ${ }^{+}$counts and higher rates of tuberculosis co-infection ( $25.3 \%$ v. $16.9 \% ; p=0.01)$. Median time to virological suppression was 29 weeks in PHC-ART initiates and 44 weeks in children down-referred $(p<0.0001)$. Children down-referred to PHC either maintained or gained virological suppression. Longitudinal cohort analysis demonstrated sustained VL suppression $>80 \%$, high rates of immune reconstitution and low mortality.

Conclusions. Increasing numbers of children are initiated on ART in PHC settings and achieve comparable immunological, virological and survival outcomes, suggesting successful decentralisation of paediatric HIV care. Down-referral of children with adherence-related virological failure may assist with attainment of virological suppression and sparing use of second-line medications.
\end{abstract}

S Afr J HIV Med 2014;15(4):148-153. DOI:10.7196/SAJHIVMED.1084

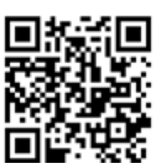

The World Health Organization (WHO) estimates that of the 2.5 million children living with HIV, $90 \%$ reside in subSaharan Africa. ${ }^{[1]}$ South Africa (SA) faces the world's largest HIV epidemic, with a staggering 330000 children affected. Perinatally acquired HIV infection is associated with high mortality. Without intervention, approximately half of all HIV-infected children will die by their second birthday ${ }^{[2-4]}$ and $35 \%$ of SA children who die before their 5 th birthday fall victim to an AIDS-related disease. ${ }^{[5]}$ More recent pooled data from southern African paediatric HIV programmes reported persistently high mortality in infants (46.3\%) despite large-scale ART roll-out. ${ }^{[6]}$

Combination antiretroviral therapy (ART) is the only effective treatment available for the suppression of HIV, and early ART initiation significantly reduces AIDS-related morbidity and mortality. ${ }^{[7,8]}$ Children in developed countries receive ART as the standard of care upon initial diagnosis; however, it is estimated that only 1 in 4 children living in subSaharan Africa receives this life-sustaining treatment. ${ }^{[1]}$ Barriers to paediatric treatment abound in subSaharan Africa: delayed diagnosis due to lack of capacity for HIV DNA polymerase chain reaction (PCR) testing, inadequate number of clinicians and staff trained to provide paediatric HIV management, and scarcity of paediatric ART drug formulations, among others. ${ }^{[9-11]}$

The WHO has recommended a decentralised, public healthcentred approach for HIV care in resource-limited settings since $2000 .^{[12,13]}$ Owing to the high level of human resources and logistical coordination needed to maintain a paediatric clinical practice and pharmacy, paediatric ART programmes have historically been located at tertiary centres and research units. With a large ruralbased population in SA, access to urban ART treatment facilities is associated with significant direct and indirect costs.

In the Western Cape Province of SA, paediatric ART first became available to public sector patients in 2004. With expansion of diagnostic and paediatric HIV management capacity, a decentralised approach to ART delivery was achieved in Cape Town's Eastern Metropole. Government and the President's Emergency Plan For AIDS Relief (PEPFAR)-funded paediatric clinicians (from the Infectious Disease Clinic (IDC) at Tygerberg Children's Hospital (TBH)) provided support to seven primary healthcare (PHC) outreach sites: Ikwezi, Grabouw, Kraaifontein, and Delft clinics; and Eerste River, Helderberg and Karl Bremer hospitals. Between 2004 and 2006, this decentralised approach to care resulted in a decrease in the number of Cape Town children 
receiving ART at tertiary centres (Red Cross War Memorial Children's Hospital, Groote Schuur Hospital and TBH) from $78.4 \%$ to $38 \% .^{[1]}$

As evidence mounts for successful initiation of paediatric ART care in rural and urban community-based PHC clinics, ${ }^{[13-18]}$ the need to evaluate paediatric down-referral outcomes remains. A systematic review of decentralised HIV care (including three studies with paediatric data) demonstrated comparable outcomes, with similar or reduced patient attrition and death at 12 months. ${ }^{[19]}$ Two large studies in subSaharan Africa demonstrated lower mortality and default rates among down-referred patients; it is notable, however, that the option of down-referral was only offered to stable, compliant patients on firstline ART. ${ }^{[20,21]} \mathrm{A}$ unique cohort of children has developed within the PHC network, as cliniciandriven longitudinal care was provided for both ART-naive initiates and those down-referred from tertiary facilities. Characterisation of this retrospective cohort not only provides evidence of the favourable long-term immunological and virological outcomes of PHC-managed children, but unique insight into the characteristics and outcomes of children who cross health-system strata through a down-referral process to community-based HIV care.

\section{Methods}

Children under 14 years of age (who were either newly initiated on ART or down-referred for continued ART management between 1 January 2004 and 30 January 2009) were identified for study inclusion from the PHC clinic registers at seven TBH-supported, community-based paediatric ART clinics. Retrospective cohort data were obtained from the IDC database (an electronic register of clinical and outcome data for children at the seven outreach clinics) to create a novel database including date of birth, first visit date at an HIV clinic, ART initiation date, ART initiation site, baseline WHO clinical stage, 6-monthly $\mathrm{CD} 4^{+}$count and viral loads (VLs). Dates of tuberculosis (TB) treatment, loss to follow-up and deaths were also extracted. Missing data were retrieved from the National Health Laboratory Service (NHLS) server or through clinic-based record reviews. Patients identified without follow-up data (as a result of ART discontinuation, transfer in or transfer out, death or loss to follow-up within 6 months of arrival in the PHC network) were categorised as 'baseline'; those with at least 6 months of follow-up data within the PHC network were assigned to the 'longitudinal' cohort.

Serial $\mathrm{CD}^{+}$counts and HIV VLs were ordered by physicians at 6-month intervals as part of standard HIV-care protocols in the Western Cape. Available laboratory data (within 3 months of each 6-monthly visit postART initiation) were included in the database. All laboratory testing was performed by the NHLS with public funds. Within the NHLS lab, $\mathrm{CD} 4^{+}$percentages are measured through the application of a dual-platform, dual-colour PanLeucogating CD45-assisted flow cytometry machine. ${ }^{[22]}$ VLs were initially measured using the NucliSens HIV-1 QT assay (bio-Merieux, Boxtel, The Netherlands), which yielded VLs undetectable below 400 copies $/ \mathrm{mL}$. Advances in sensitivity with the introduction of the NucliSens EasyQ HIV-q assay allowed more precise quantification of low-level viraemia; however, all VL values less than 400 copies $/ \mathrm{mL}$ were treated as 'suppressed'.

Clinically significant virological failure (VF) was defined as two sequential VL values $>1000$ copies $/ \mathrm{mL}$. Once patients with VF were identified, paediatric clinicians were asked to provide a clinical vignette describing adherence issues or the presumed underlying cause for VF. Maximal mortality was defined as documented deaths in addition to those lost to follow-up (LTFU). Survival analyses were calculated using the time to actual event or date of last evaluation.

The research ethics committees at Stellenbosch University and the University of Cape Town provided approval of this project. Additionally, permission to access patient health information was granted by the DeputyDirector General of District Health Services and Programmes within the Provincial Government of the Western Cape's Department of Health.

\section{Statistical analysis}

Data were compiled into Excel spreadsheets and uploaded to Stata Statistical Software

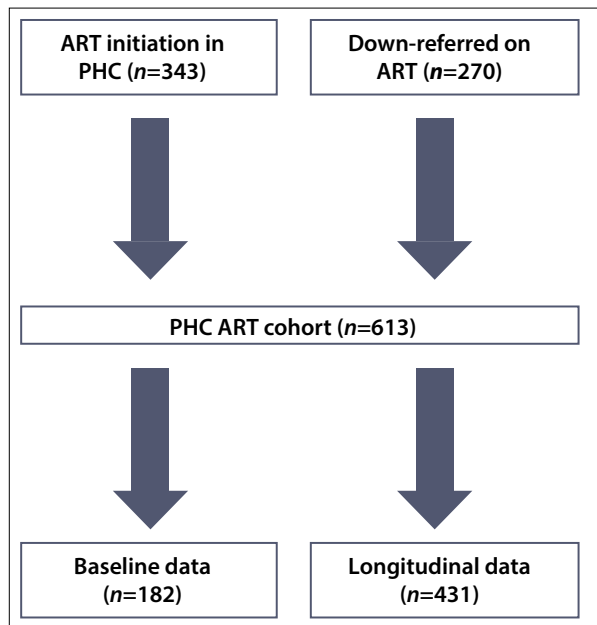

Ineligible PHC patients $(n=235)$

$153(65 \%)=$ HIV-infected, not on ART $68(29 \%)=$ dates of care in PHC not eligible by inclusion criteria

$7(3 \%)=$ significant missing data

$5(2 \%)=$ PHC not medical home

$2(1 \%)=$ HIV-exposed, uninfected

Fig. 1. Study profile. $(A R T=$ antiretroviral therapy; $P H C=$ primary healthcare. $)$ 
Table 1. PHC ART cohort characteristics stratified by ART initiation site

\begin{tabular}{|c|c|c|c|c|}
\hline Variable & $\begin{array}{l}\text { Total PHC cohort } \\
(N=613)\end{array}$ & $\begin{array}{l}\text { ART initiation in PHC } \\
(n=343)\end{array}$ & $\begin{array}{l}\text { Down-referred on ART } \\
(n=270)\end{array}$ & $p$-value \\
\hline Gender (male) (\%) & 46 & 48 & 44 & 0.358 \\
\hline Age at ART start (months), median (IQR) & $\begin{array}{l}26.4 \\
(10.2-63)\end{array}$ & $\begin{array}{l}29.6 \\
(10.0-68.4)\end{array}$ & $\begin{array}{l}25.1 \\
(10.5-59.3)\end{array}$ & 0.386 \\
\hline \multicolumn{5}{|l|}{ WHO clinical stage (\%) } \\
\hline $\mathrm{I}$ & 7.0 & 10.0 & 2.9 & 0.0009 \\
\hline II & 14.2 & 19.2 & 7.4 & 0.0001 \\
\hline III/IV (severe) & 78.9 & 70.7 & 89.8 & $<0.0001$ \\
\hline Baseline $\mathrm{CD}^{+}($cells $/ \mu \mathrm{L})$, median (IQR) & $619(323-1119)$ & $608(313-1151)$ & $650(340-1051)$ & 0.879 \\
\hline Baseline $\mathrm{CD}_{4}^{+}$percentage, median (IQR) & $16.7(10.7-23.3)$ & $17.8(11.0-24.2)$ & $16(10.0-21.8)$ & 0.016 \\
\hline \multicolumn{5}{|l|}{ Immunological category (\%) } \\
\hline No IS & 22.5 & 19.0 & 27.0 & 0.017 \\
\hline Mild IS & 18.0 & 21.0 & 14.1 & 0.026 \\
\hline Advanced IS & 21.7 & 23.6 & 19.3 & 0.194 \\
\hline Severe IS & 37.9 & 36.4 & 39.6 & 0.419 \\
\hline Baseline VL (copies/mL), median (IQR) & $\begin{array}{l}270000(49182- \\
1000000)\end{array}$ & $\begin{array}{l}230000(32000- \\
830000)\end{array}$ & $\begin{array}{l}326969(87841- \\
1554457)\end{array}$ & 0.0004 \\
\hline Baseline VL $\log \left(\log _{10}\right.$ copies $\left./ \mathrm{mL}\right)$, median (IQR) & $5.4(4.7-6.0)$ & $5.4(4.4-6.0)$ & $5.5(4.8-6.0)$ & 0.0005 \\
\hline TB at ART initiation (\%) & 20.5 & 16.9 & 25.3 & 0.013 \\
\hline
\end{tabular}

existed within the PHC network: those who were initiated on ART at higher levels of care and were down-referred to PHC, and those who were initiated on ART in PHC. The majority of those ineligible for analysis were HIV-infected patients who had not been initiated on ART before the end of our study period (Fig. 1).

Table 1 lists ART cohort characteristics stratified by ART initiation site. Baseline illness severity at the time of ART initiation, as measured by WHO clinical categorisation, was significantly different between initiation sites; proportionally more severely ill children started ART at higher levels of care than at PHC clinics $(p<0.0001)$. Additionally, those who were downreferred had significantly lower baseline $\mathrm{CD} 4^{+}$ percentages $(p=0.01)$, higher VLs $(p=0.0004)$ and greater likelihood of TB co-infection at the time ART was initiated $(p=0.01)$.

Similar trends in increased clinical severity $(p<0.0001)$, higher baseline VL $(p=0.01)$ and greater proportions of asymptomatic infants initiated on ART exist among those whose data were available for longitudinal analysis. Owing to the intensified ART roll-out in the latter half of the study period, the baseline cohort had a larger proportion of PHC-initiated patients, while the longitudinal cohort was skewed towards those down-referred $(p<0.0001)$ (Fig. 2). The characteristics and demographics of the baseline data group were therefore largely

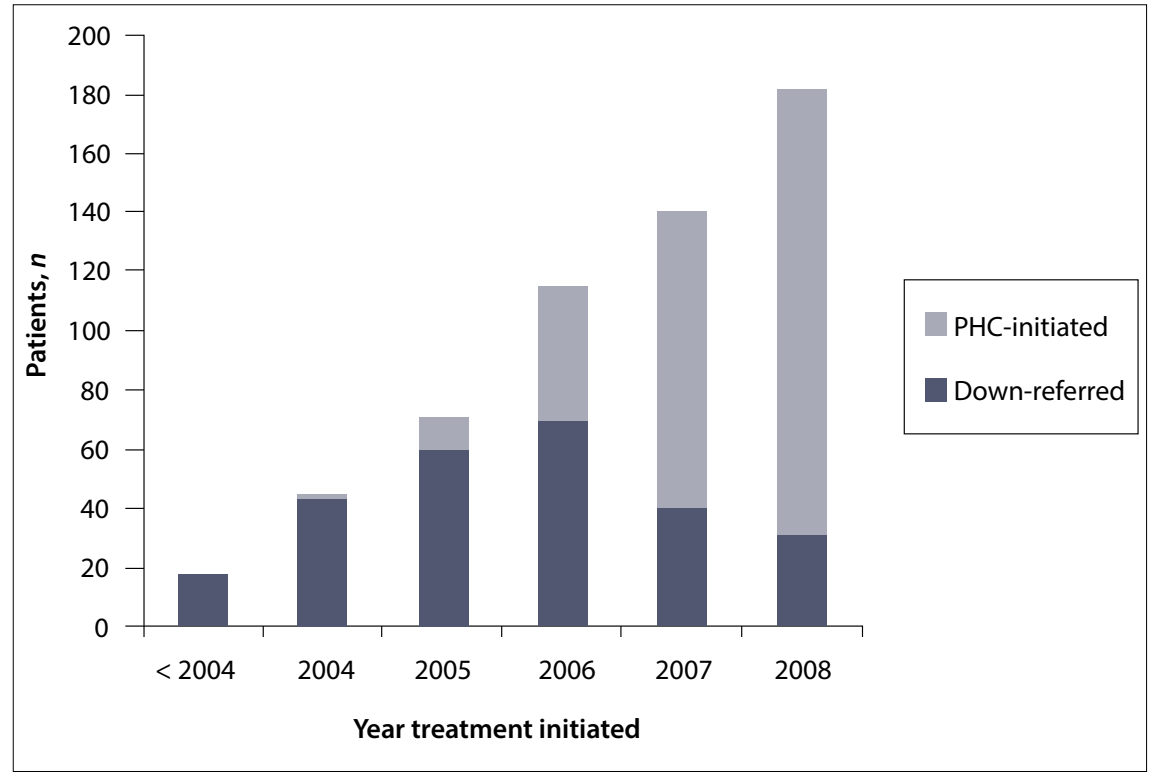

Fig. 2. $P H C$ cohort ART initiation by year and site. $(P H C=$ primary healthcare $)$

reflective of a recently initiated, lower-acuity group who initiated ART in PHC. Conversely, children in the longitudinal group were more likely to be severely ill at the time of ART initiation, and by definition had been ARTexposed for a longer period of time.

Overall, the majority of children prescribed ART had laboratory evidence of advanced or severe immunosuppression. Those without immunosuppression, however, were significantly more likely to have initiated ART outside of the PHC system $(p=0.017)$. Subanalysis confirmed increasing implementation of the revised WHO recommendations (based on the CHER trial results), ${ }^{[24]}$ which recommend ART initiation in infancy $(p=0.026)$.

\section{Virological outcomes}

The median duration of follow-up was 28 months (interquartile range (IQR) 16.5 - 42.6) 
including $>6$ years of available data for some children and a total of 1206 observed childyears. Sustained high levels of VL suppression were achieved at each time point (Fig. 3), with $>80 \%$ of the cohort suppressed from the 12 -month visit. At the last VL measurement available during the study period, $85 \%$ of the longitudinal cohort was suppressed.

Kaplan-Meier survival analysis revealed a statistically significant difference in median time to VL suppression by ART initiation site: ARTnaive patients whose care was initiated in PHC had a median time to suppression of 29 weeks, while those down-referred required 44 weeks $(p<0.0001)$. Stratification by sex, age, baseline WHO immune categorisation, modified SA WHO clinical stage and TB co-infection were not found to influence time to VL suppression.

Sixty patients $(13.9 \%$ of the group with longitudinal data; 60/431) met the criteria for clinically significant VF, with an incidence of 5.4 cases per 100 child-years. Multiple logistic regression modelling revealed that patients who started ART in the PHC system were $66 \%$ (95\% confidence interval (CI) 31 - 84) less likely to develop VF than patients who were down-referred after ART initiation in hospital. Additionally, for every one log increase in baseline VL, the odds of developing VF doubled (95\% CI 1.3 - 3.2). Age, history of TB disease, protease inhibitor-based ART regimen with TB treatment, and baseline immune category were not found to be predictive of VF within the model; baseline WHO clinical classification, however, was so significantly predictive that the variable was restricted from the model because of colinearity.

\section{Immunological outcomes}

Over $80 \%$ of the longitudinal cohort maintained a $\mathrm{CD}^{+}$percentage above baseline at each sequential monitoring period; a peak prevalence of $96 \%$ was achieved at 36 months of ART (Fig. 3). The average percentage improvement over baseline $\mathrm{CD} 4^{+}$percentage steadily rose from a median of $8.7 \%$ (IQR 2.3 $13.8)$ at 6 months to a mean of $17.4 \%(95 \%$ CI 15.5 - 19.2) at 36 months. Additionally, the largest proportion of those with $>10 \%$ improvement in $\mathrm{CD}^{+}$percentage above baseline was achieved at 36 months. Although $\mathrm{CD}^{+}$percentage gains drop off after 3 years of therapy, lower age-related norms over time are the most likely explanation for this finding rather than poor response to therapy (given rising VL suppression rates).

Thirteen children (2\% of the cohort; 1 per 100 child-years) had evidence of immunological failure (IF) after at least 24 weeks of adherent ART treatment. Delayed immune reconstitution occurred in five IF children (38.5\%); in this subgroup, $\mathrm{CD}^{+}$counts were recovered in $80 \%$ by 12 months and in all children by 18 months of ART. An additional five children (38.5\%) experienced transient IF despite concurrent VL suppression. Although IF resolved by the subsequent interval evaluation, it should be noted that $40 \%$ were undergoing concurrent TB treatment. It is therefore possible that intercurrent illnesses, TB or otherwise, may have contributed to IF. The remaining three children with IF (23\%) had persistent failure despite ongoing VL suppression. One persistent IF case experienced immune reconstitution after changing the protease inhibitor in her ART regimen from ritonavir to lopinavir/ ritonavir.

\section{Mortality}

Documented mortality among the PHC cohort was $2.2 \%$, with a maximal mortality estimate (documented deaths and LTFU cases) of 6.2\%. A disproportionate number of children were LTFU in the PHC-initiated group ( $p=0.028$ ); given the predominance of new ART initiates in this cohort, this finding was of concern for undocumented early death after starting ART (Fig. 4). Indeed, the incidence of maximal mortality was significantly higher $(p<0.0001)$ among those who received care for $<6$ months in the PHC system (183 v. 45 deaths per 10000 child-years).

A skewed temporal distribution of documented deaths was noted, with near-equal numbers experiencing early ( $<3$ months) and late (7 - 30 months) mortality. Early deaths occurred in infants $<6$ months of age, and young children (11 15 months old) with severe immunological suppression $\left(\mathrm{CD}^{+}\right.$percentage $\left.3-8.2 \%\right)$. Over half of the early mortality group (57\%) died within the first 9 days of ART therapy. Of those with early mortality, $43 \%$ were receiving dual therapy for $\mathrm{HIV}$ and $\mathrm{TB}$,

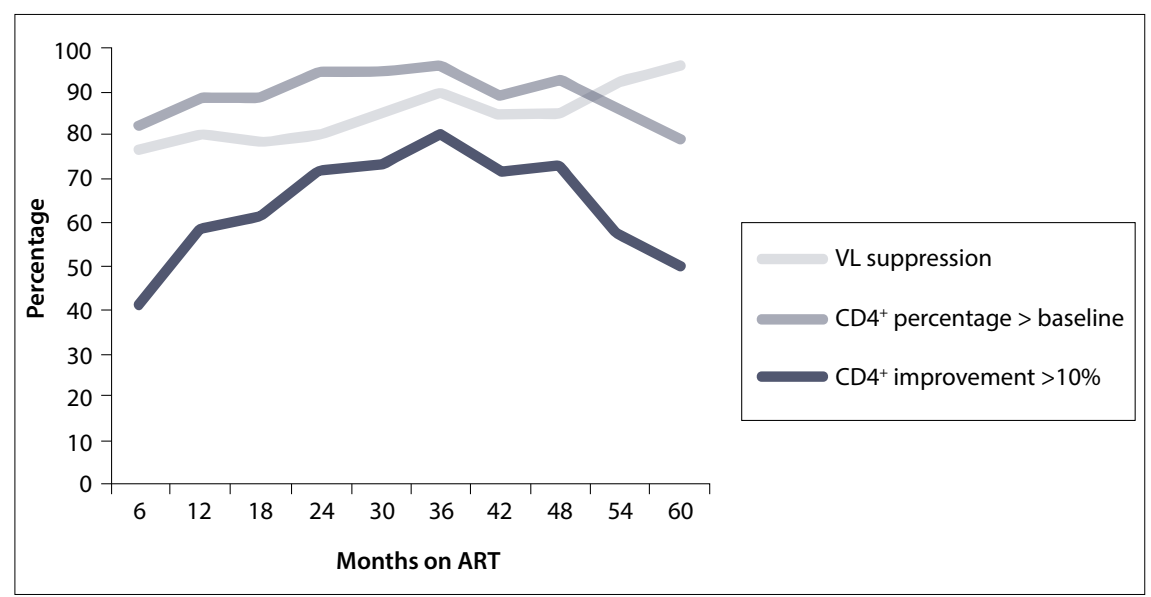

Fig. 3. Virological and immunological outcomes over 5 years of ART. (ART = antiretroviral therapy; $V L=$ viral load.)

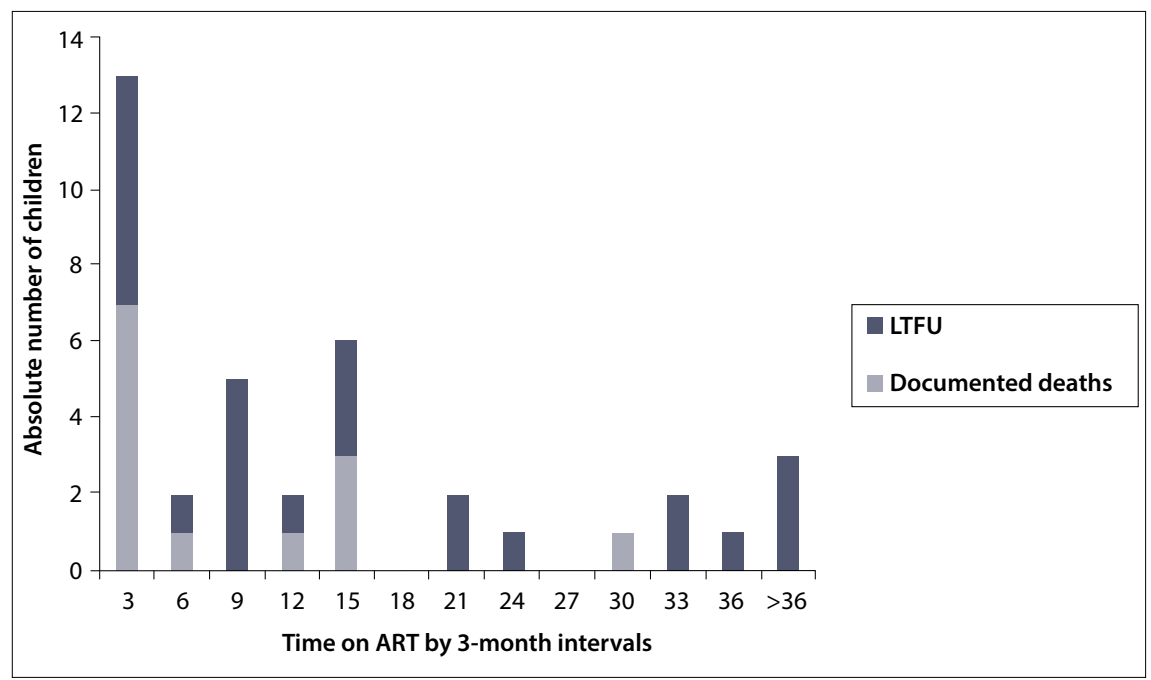

Fig. 4. Maximal mortality after ART initiation. (ART = antiretroviral therapy; LTFU = lost to follow-up.) 
with TB treatment initiated from 1 week to 3 months before ART initiation.

No difference in maximal mortality between ART initiation sites was noted once children had been on ART in the PHC clinics for at least 6 months $(p=0.09)$. Of note, early mortality data for down-referred patients were not available as a result of the nature of the study design. Four children who died were not eligible for inclusion in this analysis as they died before starting ART within the PHC network.

\section{Outcomes among children down-referred from tertiary hospitals}

After a median of 2 years (IQR 13.6 - 34 months) on ART, 153 children were down-referred from tertiary care to PHC. Of these children, $80 \%$ had a suppressed VL at the time of down-referral, and $96 \%$ of these patients remained suppressed at the last measured study evaluation. Of the 26 patients transferred with subtherapeutic response to ART, 77\% achieved virological suppression after 6 months of support and treatment within the PHC system. Three-quarters of the newly suppressed individuals met our criteria for $\mathrm{VF}$ at the time of transfer, yet only one-third of these children required medication change to second-line therapy to achieve successful suppression. Of the remaining 6 patients (23\%) with ongoing VF: 2 were changed to 3TC monotherapy (as a holding strategy); 1 was started on second-line therapy immediately prior to the end of the study period (so response to the intervention could not be assessed); 2 were transferred to the adult ART programme within the same clinic; and only 1 patient required referral back to the tertiary care centre. One down-referred child died within 3 months of transfer from a tertiary care site $(0.6 \%)$.

\section{Discussion}

Cohort characteristics reveal that ART initiation site strongly correlates with WHO clinical severity; this finding suggests appropriate triage of children with advanced HIV disease to higher levels of care. Absolute numbers of children initiated on ART within the PHC network suggest successful ART roll-out strategies for paediatric patients in the Cape Town region. Children receiving ongoing treatment within the PHC network demonstrated sustained indices of effective HIV management (high VL suppression rates, rapid immune reconstitution, low mortality rates) which are comparable with, if not better than, those in previously published studies of similar cohorts. ${ }^{[17,25-29]}$ Overall mortality in the cohort was low. Children who died exhibited the following high-risk characteristics: dual HIV/TB disease; young age ( $<6$ months); recent ART initiation ( $<3$ months on ART); and severe immunological suppression in infants $<15$ months of age. Similar trends in mortality have been noted in numerous studies, ${ }^{[18,28,3,3,31]}$ providing further evidence of a focused time period among high-risk patients who may be ideal for a targeted intervention. Intensified surveillance of this population would ideally result in expedited referral to higher levels of care and decreased mortality.

Although infants and young children are at higher risk for early mortality after ART initiation, evidence indicates that intervention before 12 weeks of age reduces this risk by $76 \% .{ }^{[24]}$ There was early adoption of Children with HIV Early Antiretroviral Therapy (CHER)-trial recommendations regarding early ART initiation (TBH was a CHER trial site), with a disproportionate number of nonimmunosuppressed infants starting ART in tertiary care. Although data to support asymptomatic infant ART initiation were first presented in mid-2007 at the International AIDS Conference on HIV Pathogenesis, Treatment and Prevention, recommendations were more widely disseminated through journal and WHO publications towards the end of the study period in 2008. ${ }^{[24,32]}$ Although there is evidence of early adoption of infant roll-out in tertiary facilities, the temporal nature of this study in respect of rapidly changing practice guidelines did not allow for true compliance evaluation. An updated analysis of interval ART initiation data by site would be required to provide an accurate assessment of infant ART roll-out and compliance with current recommendations.

Data from the PHC network are unique in that they arise from an exclusively paediatric cohort with down-referral longitudinal data. Nearperfect rates of sustained VL suppression after tertiary-to-PHC downreferral are demonstrated among stable patients. Optimistic outcomes among predominantly adult down-referred patients are congruent with our findings; however, these studies probably suffer from selection bias (those offered down-referral were preselected for success). ${ }^{[20,21]}$ Unlike these studies, 1 out of every 5 down-referred children had an unsuppressed VL at the time of transfer. Among the unsuppressed, many had VF, with clinicians noting adherence issues in most. Although down-referred children had more cumulative ART exposure with associated risk for resistance development, a high proportion achieved viral suppression after transfer to PHC without ART regimen change. Successful suppression on first-line therapy for those previously failing indicates improved adherence, likely to be secondary to decreased barriers to community-based care. PHC physicians demonstrated expertise in effectively managing those requiring second-line therapy with rare referral back to tertiary care centres and low documented mortality rates. Substantial improvement in VL suppression rates among this subgroup was observed with respect to whether a focus on adherence or a change to second-line therapy was prescribed, thereby validating the efficacy of this model of care for all-comers.

\section{Conclusion}

Although the majority of children were severely ill at ART initiation, increasing numbers of less acutely unwell children initiated ART in PHC. Our data suggested that intensified surveillance of children $<15$ months of age for the first 3 months of ART may improve mortality statistics. Reproducibly well-defined, high-risk patient characteristics restricted to a discrete high-risk period yield a clear target for programmatic intervention. Long-term ART management within PHC clinics by paediatric clinicians yields successful outcomes. Down-referral of tertiary-centre-enrolled patients with VF, probably adherence related, may be a first-line ART-sparing strategy.

Acknowledgements. M M Morsheimer and A Dramowski were supported by the National Institutes of Health Office of the Director, Fogarty International Centre, through the International Clinical Research Fellows Program at Vanderbilt University (R24 TW007988). The authors thank Prof. Landon Myer for his contributions to the data analysis and the PHC facilities' staff and patients for their contribution to and assistance with the study.

\footnotetext{
References

1. Joint United Nations Programme on HIV/AIDS (UNAIDS). Global report: UNAIDS Report on the Global AIDS Epidemic 2010. 2010. http://www.unaids.org/ globalreport/ (accessed 25 August 2014).

2. Dabis F, Elenga N, Meda N, et al. 18-Month mortality and perinatal exposure to zidovudine in West Africa. AIDS 2001;15(6):771-779. [http://dx.doi. org/10.1097/00002030-200104130-00013]
} 
3. Dunn D. HIV Paediatric Prognostic Markers Collaborative Study Group. Short-term risk of disease progression in HIV-1-infected children receiving no antiretroviral therapy or zidovudine monotherapy: A meta-analysis. Lancet 2003;362:1605-1611.

4. Spira R, Lepage P, Msellati P, et al. Natural history of human immunodeficiency virus type 1 infection in children: A five-year prospective study in Rwanda. Mother-to-Child HIV-1 Transmission Study Group. Paediatrics. 1999;104(5):e56.

5. Shisana O, Simbayi L, Rehle T, Zungu N, Zuma K, Ngogo N. South African National HIV Prevalence, Incidence, Behaviour and Communication Survey, 2008: The Health of our Children. Cape Town: Human Sciences Research Council; 2010. http://www. hsrcpress.ac.za/product.php?productid=2279 (accessed 25 August 2014).

6. Davies MA, May M, Bolton-Moore C, et al. Prognosis of children with HIV-1 infection starting antiretroviral therapy in southern Africa: A collaborative analysi of treatment programs. Pediatr Infect Dis J 2014;33(6):608-616. [http://dx.doi. org/10.1097/INF.0000000000000214]

7. De Martino M, Tovo PA, Balducci M, et al. Reduction in mortality with availability of antiretroviral therapy for children with perinatal HIV-1 infection. Italian Register for HIV Infection in Children and the Italian National AIDS Registry. JAMA 2000;284:190-197. [http://dx.doi.org/10.1001/jama.284.2.190]

8. Gibb DM, Duong T, Tookey PA, et al. Decline in mortality, AIDS, and hospita admissions in perinatally HIV-1 infected children in the United Kingdom and Ireland. BMJ 2003;327:1019.[http://dx.doi.org/10.1136/bmj.327.7422.1019]

9. Balcha TT, Jeppsson A. Outcomes of antiretroviral treatment: A comparison between hospitals and health centers in Ethiopia. J Int Assoc Physicians AIDS Care (Chic) 2010;9(5):318-324. [http://dx.doi.org/10.1177/1545109710367518]

10. Fredlund VG, Nash J. How far should they walk? Increasing antiretroviral therapy access in a rural community in northern KwaZulu-Natal, South Africa. J Infect Dis 2007;196 (Suppl 3):S469-473. [http://dx.doi.org/10.1086/521115]

11. Meyers T, Moultrie H, Naidoo K, Cotton M, Eley B, Sherman G. Challenges to paediatric HIV care and treatment in South Africa. J Infect Dis 2007;196 (Supp 3):S474-481. [http://dx.doi.org/10.1086/521116]

12. Qazi SA, Muhe LM. Integrating HIV management for children into the Integrated Management of Childhood Illness guidelines. Trans $\mathrm{R}$ Soc Trop Med Hyg 2006;100:10-13. [http://dx.doi.org/10.1016/j.trstmh.2005.05.013]

13. Gilks CF, Crowley S, Ekpini R, et al. The WHO public-health approach to antiretroviral treatment against HIV in resource-limited settings. Lancet 2006;368:505-510. [http://dx.doi.org/10.1016/S0140-6736(06)69158-7]

14. Barth RE, Tempelman HA, Smelt E, Wensing AM, Hoepelman AI, Geelen SP. Long-term outcome of children receiving antiretroviral treatment in rural South Africa: Substantial virologic failure on first-line treatment. Pediatr Infect Dis 2011;30:52-56. [http://dx. doi.org/10.1097/INF.0b013e3181ed2af3]

15. Bekker LG, Myer L, Orrell C, Lawn S, Wood R. Rapid scale-up of a communitybased HIV treatment service: Programme performance over 3 consecutive years in Guguletu, South Africa. S Afr Med J 2006;96:315-320.

16. Bock P, Boulle A, White C, Osler M, Eley B. Provision of antiretroviral therapy to children within the public sector of South Africa. Trans R Soc Trop Med Hyg 2008;102:905-911. [http://dx.doi.org/10.1016/j.trstmh.2008.06.010]

17. Bolton-Moore C, Mubiana-Mbewe M, Cantrell RA, et al. Clinical outcomes and CD cell response in children receiving antiretroviral therapy at primary health care facilitie in Zambia. JAMA 2007;298:1888-1899. [http://dx.doi.org/10.1001/jama.298.16.1888]
18. Van Griensven J, De Naeyer L, Uwera J, Asiimwe A, Gazille C, Reid T. Success with antiretroviral treatment for children in Kigali, Rwanda: Experience with health center/ nurse-based care. BMC Pediatr 2008;8:39. [http://dx.doi.org/10.1186/1471-2431-8-39]

19. Kredo T, Ford N, Adeniyi FB, Garner P. Decentralising HIV treatment in lower- and middle-income countries. Cochrane Database of Systematic Reviews 2013, Issue 6. Art. No.: CD009987. [http://dx.doi.org/10.1002/14651858.CD009987.pub2]

20. Chan AK, Mateyu G, Jahn A, et al. Outcome assessment of decentralization of antiretroviral therapy provision in a rural district of Malawi using an integrated primary care model. Trop Med Int Health 2010;15 (Suppl 1):90-97. [http://dx.doi. org/10.1111/j.1365-3156.2010.02503.x]

21. Brennan AT, Long L, Maskew M, et al. Outcomes of stable HIV-positive patients down-referred from a doctor-managed antiretroviral therapy clinic to a nursemanaged primary health clinic for monitoring and treatment. AIDS 2011;25:20272036. [http://dx.doi.org/10.1097/QAD.0b013e32834b6480]

22. Glencross D, Scott LE, Jani IV, Barnett D, Janossy G. CD45-assisted PanLeucogating for accurate cost-effective dual-platform $\mathrm{CD} 4^{+} \mathrm{T}$-cell enumeration. Cytometry 2002;50:69-77. [http://dx.doi.org/10.1002/cyto.10068]

23. Davies M, Moultrie H, Eley B, et al. Virologic failure and second-line antiretroviral therapy in children in South Africa - The IeDEA Southern Africa Collaboration. I Acquir Immune Defic Syndr 2011;56:270. [http://dx.doi.org/10.1097/ QAI.0b013e3182060610

24. Violari A, Cotton MF, Gibb DM, et al. Early antiretroviral therapy and mortality among HIV-infected infants. N Engl J Med 2008;359:2233-2244. [http://dx.doi. org/10.1056/NEJMoa0800971]

25. Fatti G, Bock P, Eley B, Mothibi E, Grimwood A. Temporal trends in baseline characteristics and treatment outcomes of children starting antiretroviral treatment An analysis in four provinces in South Africa, 2004-2009. J Acquir Immune Defic Syndr 2011;58:e60-7. [http://dx.doi.org/10.1097/QAI.0b013e3182303c7e]

26. Peacock-Villada E, Richardson BA, John-Stewart GC. Post-HAART outcomes in paediatric populations: comparison of resource-limited and developed countries. Paediatrics 2011;127:e423-41. [http://dx.doi.org/10.1542/peds.2009-2701]

27. Ciaranello AL, Chang Y, Margulis AV, et al. Effectiveness of paediatric antiretroviral therapy in resource-limited settings: A systematic review and meta-analysis. Clin Infect Dis 2009;49:1915-1927. [http://dx.doi.org/10.1086/648079]

28. Sutcliffe CG, van Dijk JH, Bolton C, Persaud D, Moss WJ. Effectiveness of antiretroviral therapy among HIV-infected children in sub-Saharan Africa. Lancet Infect Dis 2008;8:477-489. [http://dx.doi.org/10.1016/S1473-3099(08)70180-4]

29. Davies MA, Keiser O, Technau K, et al. Outcomes of the South African National Antiretroviral Treatment Programme for children: the IeDEA Southern Africa collaboration. S Afr Med J 2009;99:730-737.

30. Callens SF, Shabani N, Lusiama J, et al. Mortality and associated factors after initiation of paediatric antiretroviral treatment in the Democratic Republic of the Congo. Pediatr Infect Dis J 2009;28:35-40. [http://dx.doi.org/10.1097/ INF.0b013e318184eeb9]

31. Mubiana-Mbewe M, Bolton-Moore C, Banda Y, et al. Causes of morbidity among HIV-infected children on antiretroviral therapy in primary care facilities in Lusaka, Zambia. Trop Med Int Health 2009;14:1190-1198. [http://dx.doi.org/10.1111 /j.1365-3156.2009.02360]

32. WHO. Antiretroviral therapy for HIV infection in infants and children: Towards universal access. Recommendations for a public health approach. 2010 revision. http://www.who.int/hiv/pub/guidelines/art/en/ (accessed 25 August 2014). 